\title{
A comparison of full and partial CSI opportunistic incremental relaying networks under power constraint
}

\author{
Vo Nguyen Quoc Bao, Le Quoc Cuong \\ Wireless Communication Lab. \\ Posts and Telecommunications Institute of Technology (PTIT) \\ 11 Nguyen Dinh Chieu, District 1, Ho Chi Minh City, Vietnam \\ Email: baovnq@ptithcm.edu.vn
}

Abstract: Two well-known relay selection schemes including full relay selection (FRS) or partial relay selection (PRS) have been proposed for opportunistic cooperative networks. Different from FRS where channel state information (CSI) of the two hops is used, PRS employs only the CSI of the first hop to select the best relay, which significantly reduces the complexity, thereby extending the network life-time. In this paper, the performance comparison of the opportunistic incremental relaying networks installing two relay selection schemes is reported. Numerical results show that at low signal-to-noise ratios (SNRs), FRS outperforms PRS; However, at high SNRs, both of them approach to a limit, which is determined by the quality of the direct link between the source and the destination.

Keywords: partial relay selection, full relay selection, Rayleigh channel, incremental relaying, cooperative networks.

\section{INTRODUCTION}

Recent studies have shown that significant spatial diversity gain can be achieved in a spectral-efficient manner through opportunistic cooperation among geographically distributed wireless nodes $[1,2]$. The basic premise behind opportunistic cooperative diversity is to improve the communication between the source and the destination by using the help of the best relay (among all available ones). Depending on the strategy to select the best relay, there are two types of relay selection protocols proposed in the literature: full relay selection (FRS) [1-3] and partial relay selection (PRS) [4-13]. Although limited by its offered diversity gain, partial relay selection is a promising relay selection technique for ad-hoc and sensor networks since it reduces the need of perfect time synchronization and the centralized processing approach demanded by full relay selection, thereby prolonging the network lifetime [4].

To provide some insights on the effect of relay selection schemes, in [8], $\mathrm{K}$. Jung-Bin and $\mathrm{K}$. Dongwoo compared the conventional cooperative network installed with both schemes showing that at low SNR regime, the performance of both schemes in terms of outage and average capacity is almost the same. However, at high SNR regime, their behaviors are completely different, i.e. the performance of the network with full relay selection is linearly improved with more relays meanwhile the performance improvement offered by partial relay selection is bounded.

In cooperative communiation, incremental relaying is a promising technique since it allows to improve spectral efficiency of cooperative networks by limiting the repeating phase of the relays [13-16]. However, so far there has been no published works concerning on the effect of relay selection schemes for opportunistic incremental relaying networks.

Motivated by all of the above, in this paper, for the first time, we provide the comparison between partial relay selection and full relay selection for incremental amplify-and-forward relaying networks [13, 17] where the destination will demand the help from the best relay if the direct link between the source and the destination is not sufficiently large for decoding. To facilitate the comparison, the system performance 
metric in terms of bit error rate, outage probability and spectral efficiency of both the schemes are derived. Based on the numerical results, we show that in terms of the end-to-end bit error probability (BEP), the full relay selection scheme demonstrates its advantage over the partial relay selection scheme at low SNRs. However, their reception performance is bounded by the quality of the first time slot data transmission since the average transmit power approaches infinity. The effect of power allocation between the source and relays under fixed-power constraint condition is further investigated and discussed in this paper.

The rest of this paper is organized as follows. In Section II, we introduce two relay selection schemes and channels under study. Section III shows the formulas allowing for evaluation of average BEP, outage probability and spectral efficiency of the systems. In Section IV, we compare the performance of incremental relaying networks with two relay selection schemes and contrast the simulations and the results yielded by theory. Finally, the paper is closed in Section V.

\section{SYSTEM MODEL}

An incremental relaying network where the data transmission between the source (S) and the destination (D) is possibly helped by $N$ relays denoted as $\mathrm{R}_{1}, \mathrm{R}_{2}, \ldots, \mathrm{R}_{N}$ is considered. In this paper, we assume that all nodes in the network are equipped with a single antenna and operates in half-duplex mode. All channels are assumed to be Rayleigh fading with additive white Gaussian noise.

Let $h_{\mathrm{SD}}, h_{\mathrm{SR}_{i}}$ and $h_{\mathrm{R}_{\mathrm{D}} \mathrm{D}}$ be the link coefficients between the source to the destination, the source to relay $i$ and relay $i$ to the destination, respectively. The exponentially distributed power channels are denoted by $\quad \alpha_{0}=\left|h_{\mathrm{SD}}\right|^{2}, \quad \alpha_{1, i}=\left|h_{\mathrm{SR}_{i}}\right|^{2} \quad, \quad$ and $\alpha_{2, i}=\left|h_{\mathrm{R}_{i} \mathrm{D}}\right|^{2}$ with mean values equal to $\lambda_{0}, \lambda_{1, i}$ and $\lambda_{2, i}$, respectively. Under the fixed power constraint, the source and the relays use the transmit power $P_{1}=\xi P_{\mathrm{DT}}$ and $P_{2}=(1-\xi) P_{\mathrm{DT}}$ per transmission where $P_{\mathrm{DT}}$ denotes the transmit power for the source in case of direct transmission and $\xi$ is power allocation ratio, i.e. $\xi \in(0,1]$, thus the effective signal-to-noise ratio (SNR) at the relays and the destination resulting from the transmission of the source and the relays in the first and second time slots can be written as $\gamma_{0}=P_{1} \alpha_{0} / \mathrm{N}_{0}$, $\gamma_{1, i}=P_{1} \alpha_{1, i} / \mathrm{N}_{0}$, and $\gamma_{2, i}=P_{2} \alpha_{2, i} / \mathrm{N}_{0}$, respectively, where $\mathrm{N}_{0}$ denotes the noise variance. We further define $\bar{\gamma}_{0}=E\left\{\gamma_{0}\right\}$. To simplify matters, we shall assume as does Krikidis [4] that all channel variances in each hop are equal, i.e., $E\left\{\gamma_{1, i}\right\}=\bar{\gamma}_{1}$ and $E\left\{\gamma_{2, i}\right\}=\bar{\gamma}_{2}$ for all $i$.

\section{PERFORMANCE ANALYSIS}

\section{A. Bit Error Probability}

In incremental relaying, if the direct link SNR is greater than the SNR threshold ${ }^{1}, \gamma_{t h}$, the destination will decode the message only based on the direct signal sent by the source. Otherwise, it will request the assistance from the best relay. Therefore, for both schemes, the end-to-end bit error probability can be derived by using the law of total probability [18] as

$$
\begin{aligned}
\mathrm{BEP} & =\operatorname{Pr}\left(\gamma_{0} \geq \gamma_{t h}\right) P_{D}^{(1)}+\operatorname{Pr}\left(\gamma_{0}<\gamma_{t h}\right) P_{D}^{(2)} \\
& =\exp \left(-\frac{\gamma_{t h}}{\bar{\gamma}_{0}}\right) P_{D}^{(1)}+\left[1-\exp \left(-\frac{\gamma_{t h}}{\bar{\gamma}_{0}}\right)\right] P_{D}^{(2)}
\end{aligned}
$$

where $P_{D}^{(1)}$ denotes the average BEP at the destination provided that $\gamma_{0} \geq \gamma_{t h}$ and $P_{D}^{(2)}$ denotes the average BEP at the destination conditioned on $\gamma_{0}<\gamma_{t h}$.

We are now in position to calculate $P_{D}^{(1)}$. With coherent modulation, it is well-known that the approximations or exact values for bit error probability in the presence of fading can be obtained by averaging the error rate for the additive white Gaussian noise (AWGN) channel over the probability density function (PDF) of the SNR in Rayleigh fading, namely

$$
P_{D}^{(1)}=\int_{0}^{\infty} \varphi_{M} \operatorname{erfc}\left(\sqrt{\varsigma_{M} \gamma}\right) f_{\gamma_{0} \mid \gamma_{0} \geq \gamma_{t h}}(\gamma) d \gamma
$$

where parameters $\varphi_{M}$ and $\zeta_{M}$ depend on the type of approximation and the particular form modulation

\footnotetext{
${ }^{1}$ It defines the level of acceptable performance.
} 
provided in detail in [19. Table 6.1]. For example, $\varphi_{M}=1 / 2$ and $\varsigma_{M}=1$ for BPSK and QPSK. Furthermore $\operatorname{erfc}(x)=2 Q(x \sqrt{2})$ denotes the complementary error function. Under Rayleigh fading channel, the conditional PDF of $\gamma_{0} \mid \gamma_{0} \geq \gamma_{t h}$ is derived as [18]

$$
f_{\gamma_{0} \mid \gamma_{0}>\gamma_{\text {th }}}(\gamma)=\frac{e^{\gamma_{h t} / \bar{\gamma}_{0}}}{\bar{\gamma}_{0}} e^{-\gamma / \bar{\gamma}_{0}} \text {. }
$$

Substituting (3) into (2) and taking the integral with respect to $\gamma$, we achieve the conditional bit error probability $P_{D}^{(1)}=e^{\frac{\gamma_{\text {/h }}}{\bar{\gamma}_{0}}} \Psi_{1}\left(\bar{\gamma}_{0}\right)$, where $\Psi_{1}($.$) is defined as$

$$
\begin{aligned}
& \Psi_{1}(\bar{\gamma})=\int_{\gamma_{t h}}^{\infty} \varphi_{M} \operatorname{erfc}\left(\sqrt{\varsigma_{M} \gamma}\right) \frac{1}{\bar{\gamma}} e^{-\frac{\gamma}{\bar{\gamma}}} d \gamma \\
& =\varphi_{M}\left[e^{-\frac{\gamma_{t h}}{\bar{\gamma}}} \operatorname{erfd}\left(\sqrt{\varsigma_{M} \gamma_{t h}}\right)-\sqrt{\frac{\varsigma_{M} \bar{\gamma}}{1+\varsigma_{M} \bar{\gamma}}} \operatorname{erfc}\left(\sqrt{\frac{\gamma_{t h}}{\bar{\gamma}}\left(1+\varsigma_{M} \bar{\gamma}\right)}\right)\right]
\end{aligned}
$$

Recalling that $P_{D}^{(2)}$ is the conditional bit error rate given that $\gamma_{0}<\gamma_{t h}$ and should be considered separately depending on the relay selection technique employed, i.e. partial relay selection or full relay selection.

In the partial relay selection, the relay node with the largest signal-to-noise ratio among links from the source will serve as the forwarder in the second time slot [4] while in the FRS the relay having the highest SNR composed of SNR of the two hops will be active in the second hop $[\underline{1}, \underline{2}, \underline{20}]$. To that effect, we state that a number of algorithms have been proposed in the literature for performing this task [32]. In this paper, we adopt distributed timer technique [1]. With this mechanism, all candidate nodes of the next hop do not need to exchange or feedback either global or local state information. In particular, all relays will start a timer that is inversely proportional to the first hop channel gain (for PRS) or to the equivalent channel gain of the two hops (for FRS). The timer of the relay has best channel condition will expire first and becomes the transmitter of the next hop, while the other nodes discard the packets after receiving this node's transmission.
Under amplify-and-forward mode, the end-to-end instantaneous dual-hop SNR is well-approximated in medium-to-high SNRs as []]

$$
\begin{aligned}
\beta & = \begin{cases}\frac{\beta_{1} \beta_{2}}{\beta_{1}+\beta_{2}+1}, & \text { for PRS } \\
\max _{i=1, \ldots N}\left\{\frac{\gamma_{1, i} \gamma_{2, i}}{\gamma_{1, i}+\gamma_{2, i}+1}\right\}, & \text { for FRS }\end{cases} \\
& \approx \begin{cases}\min \left(\beta_{1}, \beta_{2}\right), & \text { for PRS } \\
\max _{i=1, \ldots N}\left\{\min \left(\gamma_{1, i}, \gamma_{2, i}\right)\right\}, & \text { for FRS }\end{cases}
\end{aligned}
$$

where $\beta_{1}=\max _{i=1, \cdots, N} \gamma_{1, i}$ denotes the SNR of the link from the source to the best relay and $\beta_{2}$ is the instantaneous SNR of the link from the best relay to the destination.

In an attempt at simplifying the complexity, selection combining is adopted at the destination instead of maximal ratio combining. The resultant SNR at the output of selection combiner is mathematically written as

$$
\gamma_{\Sigma}=\max \left(\gamma_{0} \mid\left(\gamma_{0}<\gamma_{t h}\right), \beta\right)
$$

Using the order statistics and taking into account the effect of independent between two hops yields (after several elementary computation steps)

$$
f_{\gamma_{\Sigma}}(\gamma)= \begin{cases}\frac{\sum_{i=1}^{N}(-1)^{i-1}\left(\begin{array}{c}
N \\
i
\end{array}\right)\left[\frac{1}{\bar{\gamma}_{0}} e^{-\frac{\gamma}{\gamma_{\gamma}}}+\frac{1}{\chi_{i}} e^{-\frac{\gamma}{\gamma_{i}}}-\frac{1}{\mu_{i}} e^{-\frac{\gamma}{\mu_{i}}}\right]}{1-e^{\frac{\gamma}{\gamma_{h}}}}, & \gamma_{\Sigma} \leq \gamma_{t h} \\
\sum_{i=1}^{N}(-1)^{i-1}\left(\begin{array}{c}
N \\
i
\end{array}\right) \frac{1}{\chi_{i}} e^{-\frac{\gamma}{\gamma_{i}}}, & \gamma_{\Sigma}>\gamma_{t h}\end{cases}
$$

where $\mu_{i}=\left(\chi_{i}+1 / \bar{\gamma}_{0}\right)^{-1}$ with

$$
\chi_{i}= \begin{cases}\left(i / \bar{\gamma}_{1}+1 / \bar{\gamma}_{2}\right)^{-1}, & \text { for PRS } \\ \left(1 / \bar{\gamma}_{1}+1 / \bar{\gamma}_{2}\right)^{-1}, & \text { for FRS }\end{cases}
$$

Following analogous steps to $P_{D}^{(1)}$ and splitting the integral into integrals, we have

$$
\begin{aligned}
P_{D}^{(2)} & =\int_{0}^{\infty} \varphi_{M} \operatorname{erfc}\left(\sqrt{\varsigma_{M} \gamma}\right) f_{\gamma_{2}}(\gamma) d \gamma \\
& =\sum_{i=1}^{N}(-1)^{i-1}\left(\begin{array}{c}
N \\
i
\end{array}\right)\left[\frac{\Psi_{2}\left(\bar{\gamma}_{0}\right)+\Psi_{2}\left(\chi_{i}\right)-\Psi_{2}\left(\mu_{i}\right)}{1-\exp \left(-\gamma_{t h} / \bar{\gamma}_{0}\right)}+\Psi_{1}\left(\chi_{i}\right)\right]
\end{aligned}
$$


Research, Development and Application on Information and Communication Technology

where erf(.) denotes the error function. Although the BEP in (1) is valid for medium as well as high SNRs, eq. (1) hardly provides direct insights, i.e. effects of relay selection "schemes as well as combining technique on the system performance at high SNRs. Since such insights can not be directly obtained from (1), we now develop a simple BER expression valid for high SNRs. Based on the observation at high SNRs, we note that the first term in the summation of (1) is dominant leading to the vanishing of the second term, in which case, (1) simplifies to

$\mathrm{BER} \approx \operatorname{Pr}\left(\gamma_{0} \geq \gamma_{t h}\right) P_{D}^{(1)}$

$$
\begin{aligned}
& =\Psi_{1}\left(\bar{\gamma}_{0}\right) \\
& =\varphi_{M}\left[e^{-\frac{\gamma_{t h}}{\bar{\gamma}_{0}}} \operatorname{erfo}\left(\sqrt{\varsigma_{M} \gamma_{t h}}\right)-\sqrt{\frac{\varsigma_{M} \bar{\gamma}_{0}}{1+\varsigma_{M} \bar{\gamma}_{0}}} \operatorname{erfo}\left(\sqrt{\frac{\gamma_{t h}}{\bar{\gamma}_{0}}\left(1+\varsigma_{M} \bar{\gamma}_{0}\right)}\right)\right]
\end{aligned}
$$

From (9) we can immediately conclude that PRS has a behavior identical to that of FRS at high SNRs.

\section{B. Outage Probability}

Based on the PDF of $\gamma_{\Sigma}$ derived in (7), it is straightforward to obtain the end-to-end outage probability of the system as follows:

$$
\begin{aligned}
\mathrm{OP} & =\underbrace{\operatorname{Pr}\left(\gamma_{0} \geq \gamma_{t h}\right) \operatorname{Pr}\left(\gamma_{0}<\gamma_{t h}\right)}_{=0}+\operatorname{Pr}\left(\gamma_{0}<\gamma_{t h}\right) \operatorname{Pr}\left(\gamma_{\Sigma}<\gamma_{t h}\right) \\
& =\left[1-\exp \left(-\gamma_{t h} / \bar{\gamma}_{0}\right)\right] \operatorname{Pr}\left(\gamma_{\Sigma}<\gamma_{t h}\right) \\
& =\sum_{i=1}^{N}(-1)^{i-1}\left(\begin{array}{c}
N \\
i
\end{array}\right)\left[1-e^{-\frac{\gamma_{t h}}{\bar{\gamma}_{0}}}-e^{-\frac{\gamma_{t h}}{\chi_{i}}}+e^{-\frac{\gamma_{t h}}{\mu_{i}}}\right]
\end{aligned}
$$

\section{Spectral Efficiency}

Since the quality of the direct link will decide the need of the help of the relaying link, it is interesting to note that both schemes provide the same spectral efficiency as follows:

$$
\begin{aligned}
R & =\operatorname{Pr}\left(\gamma_{0} \geq \gamma_{t h}\right) r+\operatorname{Pr}\left(\gamma_{0}<\gamma_{t h}\right) r / 2 \\
& =\left[1+\exp \left(-\gamma_{t h} / \bar{\gamma}_{0}\right)\right] r / 2
\end{aligned}
$$

where $r$ denotes the average spectral efficiency of direct transmission.

\section{NUMERICAL RESULTS}

To compare the behavior of the partial and full CSI supported relaying system, we investigate their performance under the same network settings and configurations. In particular, the network with 1, 3 and 5 relays are considered and the average channel gains are set as $\lambda_{0}=1, \lambda_{1, i}=2$ and $\lambda_{2, i}=3$ for all $i$. Note that the results with full and spatial relay selection are denoted as FRS and PRS in the figures.

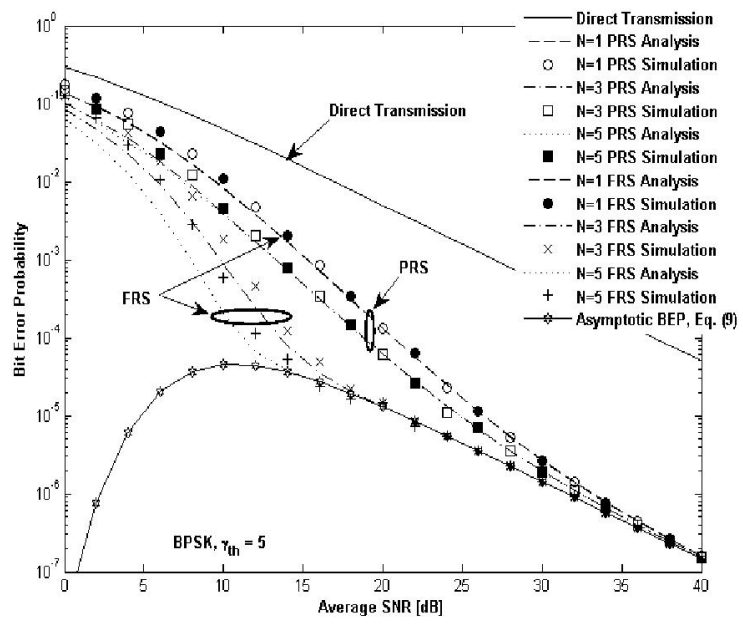

Figure 1. Effect of number of relays on BEP for BPSK.

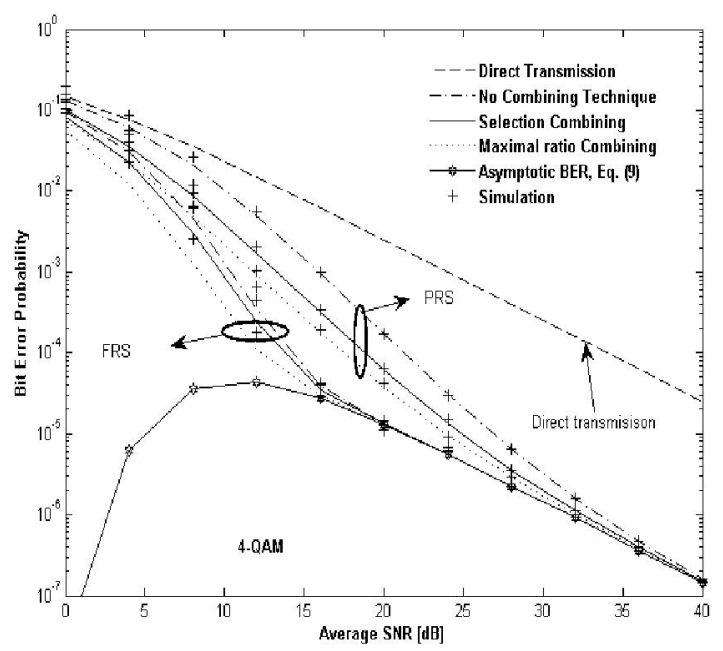

Figure 2. Effect of combining technique on BEP.

It can be observed from Fig. 1 that at low SNR regime the FRS provides significant improvement over the PRS. It also should be noted that as the number of relays $(N)$ increases, the bit error 
performance of FRS improves while that of PRS remains the same since PRS does not benefit from additional relays. However, at high SNR regime, i.e. SNR $>30 \mathrm{~dB}$, the performance of both the systems are limited by the bound. This may be explained by the fact that in such the condition, it is more likely that the quality of the direct link is good enough for decoding at the destination without the need of re-transmission from the best relay. Thus, in this case, the relay selection schemes as well as number of relays will not contribute and again will not lead to any effect on the system performance.

In Fig. 2, we investigate the effect of combining technique on the system performance. From the figure we can see that for low-to-medium average SNRs ( $\mathrm{SNR} \leq 20 \mathrm{~dB}$ ), the system with MRC outperforms that with SC, which, in turns, outperforms the system without using combining diversity. Furthermore, these observations are valid not only for PRS but also for FRS.

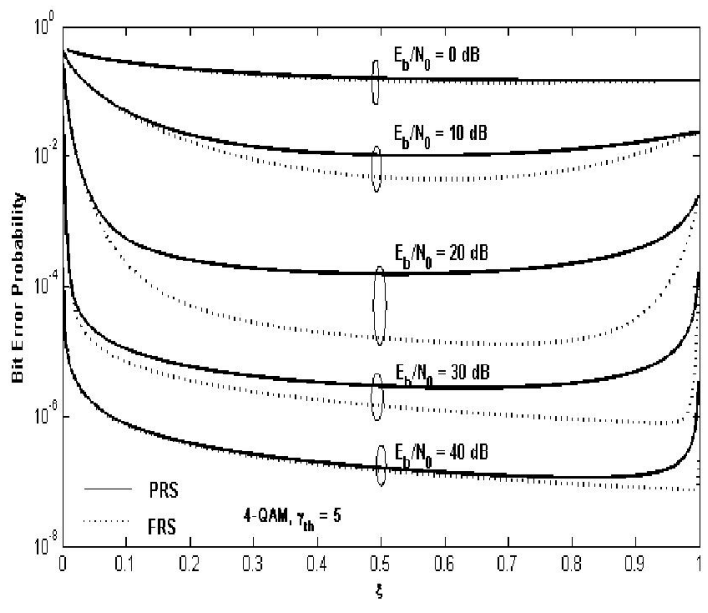

Figure 3. Effect of power allocation on BEP.

At this point, it is appropriate to comment on the power allocations under two types of relay selection. From Fig. 3, we can see that in general for incremental relaying networks it will always be better if more transmit power is allocated for the source, as expected. However, at medium SNRs, in terms of error performance, the FRS seems to be more sensitive to power allocation ratio than its counterpart.
In Fig. 4, we study the spectral efficiency of both relay selection schemes. We can see that both the relay selection schemes in increcremental networks have the same spectral effiency, which is in between that of direct transmission and that of conventional relay networks as shown in Fig. 4.

Before closing this section, it should be pointed out that in terms of outage probability, FRS always outperforms PRS under the same setting conditions as shown in Fig. 5. Furthermore, the simulation results are in good agreement with the analytical ones in high operating SNRs.

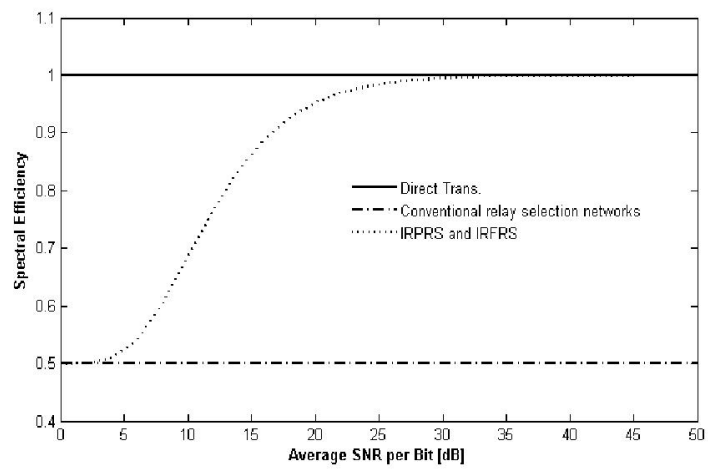

Figure 4. Spectral efficiency.

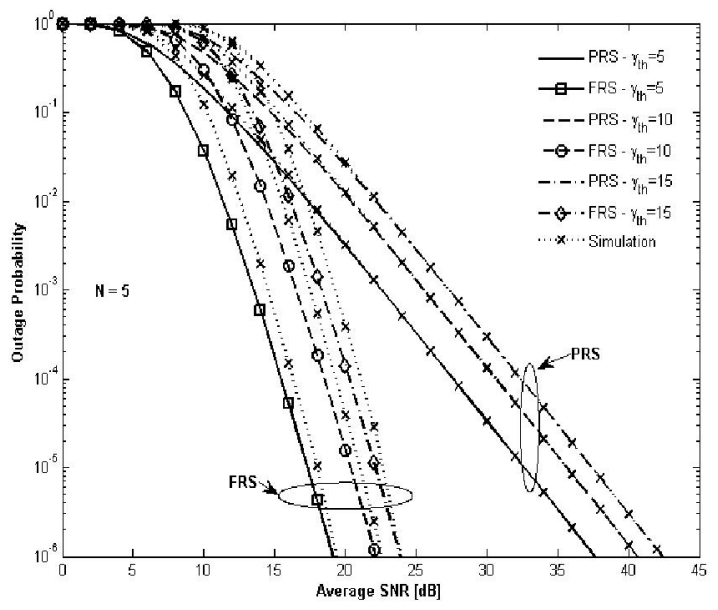

Figure 5. Effect of different relay selection schemes on $O P$. 
Research, Development and Application on Information and Communication Technology

\section{Conclusion}

We have presented a comparison of these two relay selection schemes, highlighting their features, differences, and characteristics. Specifically, the bit error probability, outage probability and achievable spectral efficiency of both schemes were derived and compared using an approximation of the effective received SNR. While it is not clear that any particular scheme is the best for all scenarios, each scheme has definite advantages and disadvantages, and is well suited for certain situations and networks.

Acknowledgment: This research was supported by the Vietnam's National Foundation for Science and Technology Development (NAFOSTED) (No. 102.99-2010.10).

\section{REFERENCES}

[1] A. Bletsas, A. Khisti, D. P. Reed, and A. Lippman, "A Simple Cooperative Diversity Method Based on Network Path Selection," IEEE Journal on Select Areas in Communications, vol. 24, pp. 659-672, March 2006.

[2] A. Bletsas, H. Shin, and M. Z. Win, "Outage Analysis for Cooperative Communication with Multiple Amplify-and-Forward Relays," Electronics Letters, vol. 43, 15th March 2007.

[3] S. S. Ikki and M. H. Ahmed, "Performance of Multiple-Relay Cooperative Diversity Systems with Best Relay Selection over Rayleigh Fading Channels," EURASIP Journal on Applied Signal Processing, vol. 2008, 2008.

[4] I. Krikidis, J. Thompson, S. McLaughlin, and N. goertz, "Amplify-and-Forward with Partial Relay Selection," IEEE Communications Letters, vol. 12, pp. 235-237, 2008.

[5] V. N. Q. Bao and H. Y. Kong, "Diversity Order Analysis of Dual-hop Relaying with Partial Relay Selection," IEICE Trans Commun, vol. E92-B, pp. 3942-3946, Dec. 2009.

[6] D. B. da Costa and S. Aissa, "End-to-End Performance of Dual-Hop Semi-Blind Relaying Systems with Partial Relay Selection," IEEE Transactions on Wireless Communications, vol. 8, pp. 4306-4315, 2009.

[7] D. B. D. Costa and S. AÂ $¿$ ssa, "Dual-hop decode-andforward relaying systems with relay selection and maximal-ratio schemes," Electronics Letters, vol. 45, pp. 460-461, 2009.

[8] K. Jung-Bin and K. Dongwoo, "Comparison of Tightly Power-Constrained Performances for Opportunistic Amplify-and-Forward Relaying with Partial or Full
Channel Information," Communications Letters, IEEE, vol. 13, pp. 100-102, 2009.

[9] H. A. Suraweera, D. S. Michalopoulos, and G. K. Karagiannidis, "Semi-blind amplify-and-forward with partial relay selection," Electronics Letters, vol. 45, pp. 317-319, 2009.

[10]H. Boujemâa, "Transmission Systems Exact symbol error probability of cooperative systems with partial relay selection," Eur. Trans. Telecomms., vol. 21, pp. $79-85,2010$.

[11]H. Ding, J. Ge, and Z. Jiang, "Asymptotic performance analysis of amplify-and-forward with partial relay selection in Rician fading," Electronics Letters, vol. 46, 4th February 2010.

[12]X. Rui, "Decode-and-forward with partial relay selection," International Journal of Communication Systems, vol. -, pp. -, 2010.

[13] V. N. Q. Bao and H. Y. Kong, "Incremental relaying for partial relay selection," IEICE Trans Commun, vol. E93-B, pp. 1317-1321, May 2010.

[14] K. Tourki, H. Yang, and M. Alouini, "Accurate Outage Analysis of Incremental Decode-and-Forward Opportunistic Relaying," Wireless Communications, IEEE Transactions on, vol. PP, pp. 1-5, 2011.

[15] V. N. Q. Bao, N. T. Duc, and H. D. Chien, "Incremental Cooperative Diversity for Wireless Networks under Opportunistic Spectrum Access," in The 2011 International Conference on Advanced Technologies for Communications, Danang, Vietnam, 2011, pp. 121-125.

[16] S. S. Ikki and M. H. Ahmed, "Performance Analysis of Decode-and-Forward Incremental Relaying Cooperative-Diversity Networks over Rayleigh Fading Channels," in Vehicular Technology Conference, 2009. VTC Spring 2009. IEEE 69th, 2009, pp. 1-6.

[17] S. Ikki and M. H. Ahmed, "PHY 50-5 - Performance Analysis of Incremental Relaying Cooperative Diversity Networks over Rayleigh Fading Channels," in Wireless Communications and Networking Conference, 2008. WCNC 2008. IEEE, 2008, pp. 1311-1315.

[18] A. Papoulis and S. U. Pillai, "Probability, random variables, and stochastic processes", 4th ed. Boston: McGraw-Hill, 2002.

[19] A. Goldsmith, "Wireless communications", Cambridge; New York: Cambridge University Press, 2005.

[20] A. Bletsas, H. Shin, and M. Z. Win, "Cooperative Communications with Outage-Optimal Opportunistic Relaying," IEEE Transactions on Wireless Communications, vol. 6, pp. 3450-3460, September 2007. 


\section{AUThors' BIOGraPhies}

\section{2}

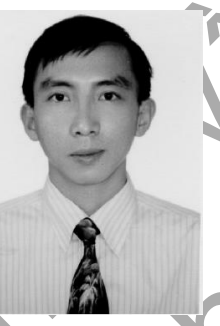

TVo Nguyen Quoc Bao received the B.E. and the M.S. degrees in Electronics and Telecommunications Engineering from Ho Chi Minh City University of Technology, Vietnam, in 2002 and 2005, respectively. And he received $\mathrm{Ph} . \mathrm{D}$. degree from University of Ulsan, Korea in 2010. Currently he is an assistant professor at School of Telecommunications, Posts and Telecommunications Institute of Technology (PTIT), Ho Chi Minh City, Vietnam. His major research interests are modulation and coding techniques, MIMO system, digital signal processing, combining technique, cooperative communications. He is a member of KICS, IEICE and IEEE. Email: baovnq@ptithcm.edu.vn

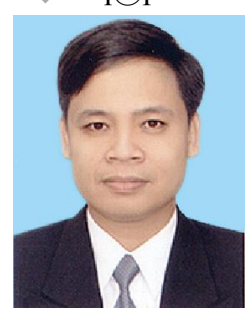

Le Quoc Cuong was born in Long An on Dec. 22, 1968. He received B.S. and $\mathrm{Ph} . \mathrm{D}$. degrees in Electronics and Telecommunications Engineering from University of Leningrat and SaintPeterburg University of Telecommunication (SUT) in 1987 and 1996, respectively. Currently, he is the vice president (in charge of Hochiminh City Campus) of Posts and Telecommunications Institute of Technology. His research interests include signal processing, optical networks and digital communications. Email: lequoccuong@ptithcm.edu.vn. 